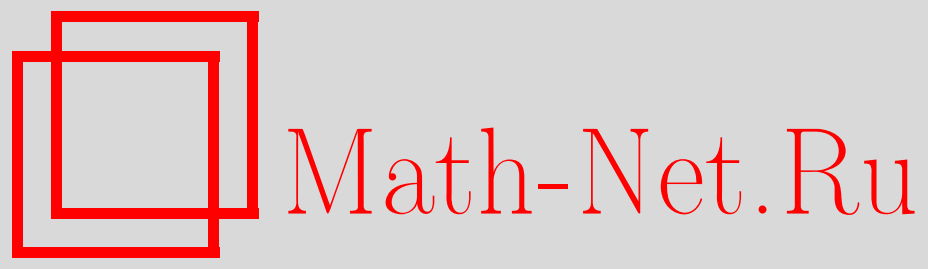

Д. А. Попов, О формуле Вейля для оператора Лапласа на гиперболических римановых поверхностях, Функи. анализ и его прил., 2014, том 48, выпуск 2, 93-96

DOI: https://doi.org/10.4213/faa3148

Использование Общероссийского математического портала MathNet.Ru подразумевает, что вы прочитали и согласны с пользовательским соглашением

http://www . mathnet.ru/rus/agreement

Параметры загрузки:

IP : 54.81 .137 .203

26 апреля 2023 г., 14:27:35

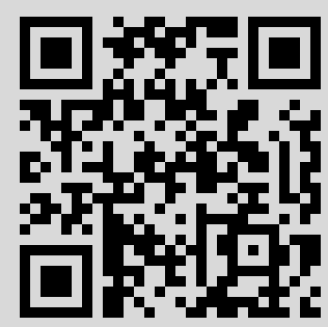


УДК 517.984.5

\title{
О формуле Вейля для оператора Лапласа на гиперболических римановых поверхностях
}

\author{
(c) 2014. Д. А. Попов
}

Памяти Израиля Моисеевича Гельфанда

1. Введение. Пусть $\Gamma$ - строго гиперболическая фуксова группа, $H-$ верхняя полуплоскость и $F=\Gamma \backslash H$ - замкнутая риманова поверхность с римановой метрикой постоянной отрицательной кривизны (метрикой Пуанкаре). Пусть $\left\{\lambda_{n}\right\}\left(\lambda_{0}=0<\lambda_{1} \leqslant \lambda_{2} \leqslant \lambda_{3} \leqslant \ldots\right)$ - спектр оператора Лапласа $\Delta$ $\left(\Delta \varphi_{n}+\lambda_{n} \varphi_{n}=0\right)$ и

$$
N(x)=\#\left\{n \geqslant 0, \lambda_{n} \leqslant x\right\}=\sum_{\lambda_{n} \leqslant x} 1
$$

- функция распределения собственных значений. В соответствии с формулой Вейля

$$
N(x)=A x+\Delta N(x), \quad|\Delta N(x)|=O\left(x^{1 / 2}\right) \quad(x \rightarrow \infty),
$$

где $A=(4 \pi)^{-1}|F|=g-1$ и $|F|-$ площадь, а $g-$ род поверхности $F$. Нас будет интересовать поведение величины $\Delta N(x)$ при $x \rightarrow \infty$. Известно [1], что $\Delta N(x)=O\left(x^{1 / 2}(\ln x)^{-1}\right)$, и имеется гипотеза, что для группы $\Gamma$, отвечающей точке общего положения в пространстве Тейхмюллера, $|\Delta N(x)|=O\left(x^{\varepsilon}\right)$ для любого $\varepsilon>0$ (см. [2], [3]). С другой стороны, Сельберг доказал, что для некоторой бесконечной серии арифметических групп $|\Delta N(x)|=\Omega\left(x^{1 / 4}(\ln x)^{-1}\right)$ (см. [4]), т. е. существует последовательность $x_{i} \rightarrow \infty$, такая, что $\left|\Delta N\left(x_{i}\right)\right|>$ $C x_{i}^{1 / 4}\left(\ln x_{i}\right)^{-1}$. Для любой группы рассматриваемого типа известно, что $|\Delta N(x)|$ $=\Omega\left((\ln x / \ln \ln x)^{1 / 2}\right)$. Заметим, что, как показано в [5],

$$
X^{-1} \int_{0}^{X}|\Delta N(x)| d x=O\left(X^{1 / 4+\varepsilon}\right) .
$$

Вместо $\lambda_{n}$ удобно рассматривать величины $r_{n}$, определенные равенствами

$$
\lambda_{n}=r_{n}^{2}+1 / 4 .
$$

Цель настоящей работы - сформулировать условия на распределение величин $r_{n}$, достаточные для существования степенного понижения в формуле Вейля, т. е. для справедливости оценки

$$
|\Delta N(x)|=O\left(x^{\theta}\right), \quad \theta<1 / 2 .
$$

Насколько мне известно, такая оценка до сих пор не доказана.

2. Основные результаты. Пусть $P-$ класс сопряженных в $Г$ элементов, $N(P)$ - его норма [4],

$$
B_{0}=\min _{\{P\}}\{N(P)\}, \quad b_{0}=\ln B_{0} \quad\left(b_{0}>0\right), \quad B=\left\{\begin{array}{ll}
2, & 1<B_{0}<2, \\
B_{0}, & B_{0} \geqslant 2,
\end{array} \quad b=\ln B .\right.
$$


Через $C$ ниже обозначаются константы, зависящие только от группы $\Gamma$, и их явный вид может быть указан.

Используя теорему 1 и следствие 1 из работы [7] и следуя рассуждениям работы [6], получаем следующий результат.

Теорема 1. Пусть Г - произвольная строго гиперболическая группа. Тогда для любого $t \geqslant 3, t \notin\left\{r_{n}\right\}$,

$$
N\left(t^{2}+1 / 4\right)=\frac{|F|}{4 \pi} t^{2}-\frac{1}{\pi} S(t)+F(t) .
$$

В этой формуле

$$
\begin{aligned}
S(t) & =\sum_{r_{n} \geqslant 0}\left[\operatorname{sgn}\left(r_{n}-t\right) \operatorname{si}\left(\left|t-r_{n}\right| b\right)-\operatorname{si}\left(\left(t+r_{n}\right) b\right)-\beta_{n}(t)\right], \\
\beta_{n}(t) & =-\frac{r_{n} \cos \left(r_{n}-t\right) b}{b\left(r_{n}^{2}+1 / 4\right)}+\frac{1}{2} \frac{\sin \left(r_{n}-t\right) b}{b\left(r_{n}^{2}+1 / 4\right)}+\frac{r_{n} \cos \left(r_{n}+t\right) b}{b\left(r_{n}^{2}+1 / 4\right)}-\frac{1}{2} \frac{\sin \left(r_{n}+t\right) b}{b\left(r_{n}^{2}+1 / 4\right)},
\end{aligned}
$$

и $F$ - ограниченная непрерывная функиия, имеющая при $t \rightarrow \infty$ асимптотику вида

$$
F(t)=C_{0}+\frac{C_{1}}{t}+\frac{C_{2}}{t^{2}}+\cdots .
$$

Здесь использовано стандартное обозначение si(•) - интегральный синус,

$$
\text { si } x=\int_{-\infty}^{x} \frac{\sin t}{t} d t .
$$

Эта теорема является обобщением результата работы [6] на любые строго гиперболические группы. Сходимость ряда $S(t)$ при $t \neq r_{n}$ следует из формулы Сельберга и доказывается так же, как в работе [6]. При этом остаются верными все утверждения работ [6], [7] о характере сходимости этого ряда. Кроме того, можно показать, что

$$
\sum_{r_{n} \geqslant R} \frac{\cos r_{n} b}{r_{n}^{2}}=O\left(\frac{1}{R}\right) \quad(R \rightarrow \infty) .
$$

Везде ниже предполагается, что $t \geqslant t_{0}$, где $t_{0}-$ некоторая достаточно большая величина, которая может быть явно указана. Это условие будет записываться в виде $t \gg 1$. Это относится и к другим величинам, т. е. к условиям вида $\xi \gg 1$. Запись $f(t)=O(\varphi(t))$ означает, что $|f(t)| \leqslant C \varphi(t)$ при $t \gg 1$.

Рассмотрим сумму

$$
\Phi(\xi)=\sum_{0 \leqslant r_{n} \leqslant \xi} e^{i r_{n} b} \quad(\xi \gg 1)
$$

и будем писать $\left\{r_{n}\right\} \in \Pi_{\alpha}$, если существует $\alpha=\alpha_{\Gamma}, 0<\alpha \leqslant 1$, такое, что

$$
|\Phi(\xi)| \leqslant C \xi^{2 \alpha} \quad(\xi \gg 1) .
$$

При $\alpha=1$ это условие заведомо выполняется, так как

$$
|\Phi(\xi)| \leqslant \sum_{0 \leqslant r_{n} \leqslant \xi} 1 \leqslant C \xi^{2},
$$

причем последнее неравенство следует из формулы Вейля (1). 
Заметим, что в настоящее время не известны примеры групп, для которых доказано, что $\left\{r_{n}\right\} \in \Pi_{\alpha}$ при $\alpha<1$.

Лемма 1. Если $t \gg 1, \delta=t^{\gamma}, 0<\gamma<1, u\left\{r_{n}\right\} \in \Pi_{\alpha}$, то для любъх таких $\alpha u \gamma$

$$
S(t)=Q_{\delta}(t)+P_{\delta}(t)+S_{\delta}^{0}(t)+O\left(\delta+t^{2 \alpha} / \delta^{3}\right) .
$$

$B$ этой формуле величина $Q_{\delta}(t)$ определяется равенством

$$
\begin{aligned}
Q_{\delta}(t)=\frac{t \cos t b}{b} \sum_{\left|r_{n}-t\right| \geqslant \delta}^{\prime} \frac{\cos r_{n} b}{r_{n}} & \left(\frac{1}{r_{n}-t}+\frac{1}{r_{n}+t}\right) \\
& +\frac{t \sin t b}{b} \sum_{\left|r_{n}-t\right| \geqslant \delta}^{\prime} \frac{\sin r_{n} b}{r_{n}}\left(\frac{1}{r_{n}-t}-\frac{1}{r_{n}+t}\right),
\end{aligned}
$$

величина $P_{\delta}(t)$ - равенством

$$
\begin{aligned}
P_{\delta}(t)=\frac{\cos t b}{b^{2}} \sum_{\left|r_{n}-t\right| \geqslant \delta}^{\prime} & \sin r_{n} b\left(\frac{1}{\left(r_{n}-t\right)^{2}}-\frac{1}{\left(r_{n}+t\right)^{2}}\right) \\
& -\frac{\sin t b}{b^{2}} \sum_{\left|r_{n}-t\right| \geqslant \delta}^{\prime} \cos r_{n} b\left(\frac{1}{\left(r_{n}-t\right)^{2}}+\frac{1}{\left(r_{n}+t\right)^{2}}+\frac{b}{r_{n}^{2}}\right)
\end{aligned}
$$

u $S_{\delta}^{0}(t)-$ равенством

$$
S_{\delta}^{0}(t)=\sum_{t-\delta<r_{n}<t} \operatorname{si}\left(t-r_{n}\right) b-\sum_{t<r_{n}<t+\delta} \operatorname{si}\left(r_{n}-t\right) b .
$$

Штрих у знака суммы означает, что суммирование ведется по области $r_{n} \geqslant 1$. Используя (5), можно показать, что если $R>2 t$, то в (10) и (11) можно перейти к конечным суммам по области $1 \leqslant r_{n} \leqslant R$ и при этом надо только заменить $O\left(\delta+t^{2 \alpha} / \delta^{3}\right)$ в правой части формулы $(9)$ на $O\left(\delta+t^{2 \alpha} / \delta^{3}+t^{2} / R\right)$.

Доказательство леммы 1 проводится прямым вычислением, исходя из формулы (4). При этом достаточно использовать оценки (7), (8), формулу частичного суммирования Абеля и тот факт, что для любого $N \geqslant 1$ при $x \geqslant 1$

$$
\begin{gathered}
-\operatorname{si} x=f_{N}(x) \cos x+g_{N}(x) \sin x+\varphi_{N}(x), \\
f_{N}(x)=\frac{1}{x}-\frac{2 !}{x^{3}}+\frac{4 !}{x^{5}}+\cdots+(-1)^{N} \frac{(2 N-2) !}{x^{2 N-1}} \\
g_{N}(x)=\frac{1}{x^{2}}-\frac{3 !}{x^{4}}+\frac{5 !}{x^{6}}+\cdots+(-1)^{N} \frac{(2 N-1) !}{x^{2 N}}, \\
\left|\varphi_{N}(x)\right| \leqslant C_{N} x^{-2 N-1} .
\end{gathered}
$$

Теорема 2. Пусть строго гиперболическая группа $\Gamma$ такова, что $\left\{r_{n}\right\} \in$ $\Pi_{\alpha}, \alpha \equiv \alpha_{\Gamma}<1, u$ nрu $\delta=t^{\alpha}$

$$
\left|S_{\delta}^{0}(t)\right| \leqslant C \delta
$$

Тогда в формуле Вейля (1)

$$
|\Delta N(x)| \leqslant C x^{\alpha / 2} \quad(x \gg 1) .
$$


Используя формулу частичного суммирования и оценку (7), получаем, что

$$
\left|P_{\delta}(t)\right| \leqslant c \frac{t^{2 \alpha}}{\delta}, \quad\left|Q_{\delta}(t)\right| \leqslant C \frac{t^{2 \alpha}}{\delta},
$$

и, с учетом того, что $x=t^{2}+1 / 4$, оценка (14) прямо следует из (3), (9). Заметим, что если в формуле (2) $\theta<1 / 2$, то $\alpha<1$. Точнее, имеет место

Лемма 2. Если справедлива оценка (2), то $\left\{r_{n}\right\} \in \Pi_{\alpha}$ и при этом

$$
\alpha=1 / 2+\theta .
$$

Доказательство леммы 2 основано на том, что из (2) следуют соотношения

$$
r_{n}=r_{n}^{0}+\Delta r_{n}, \quad\left|\Delta r_{n}\right|=O\left(\left(r_{n}^{0}\right)^{2 \theta-1}\right), \quad r_{n}^{0}=\sqrt{n / A} \quad(n \gg 1)
$$

и, таким образом,

$$
\begin{gathered}
\Phi(\xi)=\Phi_{0}(\xi)+O\left(\xi^{1+2 \theta}\right) \quad(\xi \gg 1), \\
\Phi_{0}(\xi)=\sum_{r_{0} \leqslant r_{n} \leqslant \xi} e^{i r_{n}^{0} b}=\sum_{r_{0} \leqslant r_{n} \leqslant \xi} e^{i 2 \pi f(n)}, \quad r_{0} \leqslant C \xi^{1 / 2+\theta} .
\end{gathered}
$$

Так как при $\xi \gg 1$ в интервале $\left[r_{0}, \xi\right]$ справедливо равенство $\left|f^{(1)}(x)\right|<1$ и величина $\left|f^{(2)}(x)\right|$ не меняет знак, то (см. [7]) с ошибкой $O(1)$ сумму $\Phi_{0}(x)$ можно заменить интегралом. Это сразу приводит к оценке $\left|\Phi_{0}(\xi)\right| \leqslant C \xi$. Предположим, что функция $G(R)=\sum_{r_{n} \geqslant R} e^{i r_{n} b} r_{n}^{-2}$ дифференцируема и допускает асимптотическое разложение вида $G(R)=C_{1} R^{-1}+C_{2} R^{-2}+\cdots$, которое можно почленно дифференцировать. Используя формулу Абеля, получаем, что $G^{(1)}(R)=2 \Phi(R) R^{-3}$ и, следовательно, если функция $G$ удовлетворяет указанным выше условиям, то $\alpha \leqslant 1 / 2$. В заключение отметим, что так как в сумме $\Phi(\xi)$ порядка $\xi^{2}$ слагаемых, то при $\alpha=1 / 2+\varepsilon$ эта сумма оценивается п. в. как сумма независимых случайных величин с нулевыми средними.

\section{ЛитерАтУРА}

[1] P. H. Berard, Math. Z., 155:3 (1977), 249-276. [2] Д. В. Косыгин, А. А. Минасов, Я. Г. Синай, УМН, 48:4 (1993), 3-130. [3] D. Jakobson, I. Polterovich, J. A. Toth, A lower bound for the remainder in Weyl's law on negatively curved surfaces, http://arxiv.org/abs/math/0612250. [4] D. A. Hejhal, The Selberg Trace Formula for $\operatorname{PSL}(2, \mathbb{R})$, V. 1, Lecture Notes in Math., vol. 548, Springer-Verlag, Berlin-New York, 1976. [5] В. Randol, Bull. London Math. Soc., 13 (1981), 309-315. [6] Д. А. Попов, Функц. анализ и его прил., 46:2 (2012), 66-82. [7] Д. А. Попов, Функц. анализ и его прил., 47:4 (2013), 53-66. [8] А. А. Карацуба, УМН, 45:5 (1990), 82-140.

Научно-исследовательский институт физико-химической биологии им. А. Н. Белозерского, Поступило в редакцию МГУ им. М. В. Ломоносова

Заведующая редакцией и научный редактор Г. М. Цукерман

Подписано к печати 29.04.2014. Дата выхода в свет 20.05.2014. Формат 70×100/16 Печать офсетная. Усл. печ. л. 7,8. Усл. кр.-отт. 1,4 тыс. Бум. л. 3,0 Уч.-изд. л. 8,0. Тираж 174 экз. Заказ 109.

Учредители: Российская академия наук, Математический институт им. В. А. Стеклова РАН

Адрес редакции: 117966 Москва, ГСП-1, ул. Губкина 8, комн. 624. Тел. 938-37-56

Издатель: Российская академия наук, Издательство «Наука»,

Отпечатано в ППП «Типография «Наука», 121099 Москва, Шубинский пер., 6 